\title{
A Validation of ICA Decomposition for PolSAR Images by Using Measures of Normalized Compression Distance
}

\author{
Radu TĂNASE ${ }^{(1,2)}$, Corina VĂDUVA ${ }^{(1)}$, Mihai DATCU ${ }^{(3,1)}$, Dan RĂDUCANU \\ CEOSpaceTech, University Politehnica of Bucharest, Bucharest, Romania \\ GEOINT Center, Military Technical Academy, Bucharest, Romania \\ Remote Sensing Technology Institute, German Aerospace Center, Wessling, Germany \\ radu tns@yahoo.com, corina.vaduva@gmail.com, mihai.datcu@dlr.de, dan.raducanu@gmail.com
}

\begin{abstract}

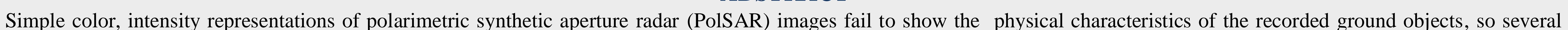

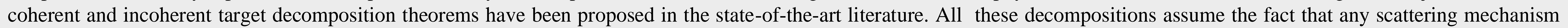

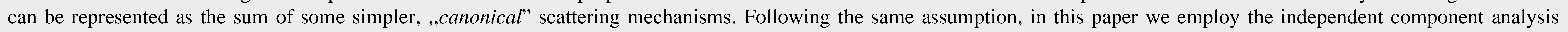

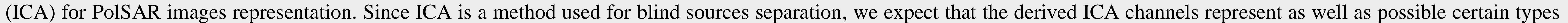

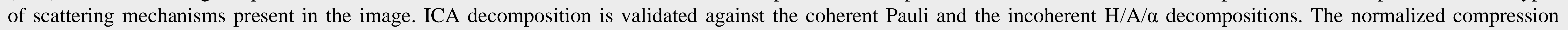
distance (NCD) is used as a measure of quality of decompositions. Experiments are made on a SLC L-band F-SAR image over Kaufbeuren airfield, Germany.
\end{abstract}

\section{The Pauli decomposition}

\section{STATE-OF-THE-ART}

The coherent target decompositions are suitable for representing ground scenes in which coherent, pure targets are predominant, A well chosen combination of the polarimetric channels contained in the scattering matrix $S$ would show the underlying physical characteristics of the ground objects;

$$
S=\left[\begin{array}{cc}
H H & H V \\
V H & V V
\end{array}\right] \quad \vec{k}=\frac{1}{\sqrt{2}} \cdot\left[\begin{array}{c}
H H+V V \\
H H-V V \\
2 H V
\end{array}\right]
$$

The three Pauli components reflect the backscattering properties of the ground objects. The first component is referred to single-bounce or odd-bounce scattering, the second component is referred to double-bounce or even-bounce scattering, while the third component corresponds to volume scattering.

\section{The H/A/ a decomposition}

When a particular pixel corresponds to a distributed scatterer, incoherent decompositions of the second order polarimetric representations (the coherency matrix $T$ ) have to be employed in order to distinguish simpler, ,canonical” scattering mechanisms.

$$
\begin{array}{ccc}
T=\vec{k} \cdot \vec{k}^{\dagger} & u_{i}=\left[\begin{array}{c}
\cos \alpha_{i} \\
\sin \alpha_{i} \cos \beta_{i} e^{j \delta_{i}} \\
\sin \alpha_{i} \cos \beta_{i} e^{j \gamma_{i}}
\end{array}\right] & H=\sum_{i=1}^{3}-p_{i} \log _{3} p_{i} \\
p_{i}=\lambda_{i} / \sum_{j=1}^{3} \lambda_{j} & \alpha=\sum_{i=1}^{3} p_{i} \alpha_{i} & A=\frac{\lambda_{2}-\lambda_{3}}{\lambda_{2}+\lambda_{3}}=\frac{p_{2}-p_{3}}{p_{2}+p_{3}}
\end{array}
$$

An $\alpha$ close to 0 corresponds to single bounce scattering, an $\alpha$ close to $\pi / 4$ corresponds to volume scattering and an $\alpha$ close to $\pi / 2$ corresponds to double bounce scattering. $H$ represents the degree of randomness of the scattering process, while $A$ can discriminate certain types of scatterers in case of high values of $\mathrm{H}$.

\section{The Independent Component Analysis}

THE PROPOSED APPROACH

ICA is a blind sources separation method used to retrieve some independent random variables (sources $-S$ ) from some linear mixtures of them (observations $-X$ ).

$$
X=A S \rightarrow \tilde{S}=\widetilde{A^{-1}} X
$$

Considering the three polarimetric channels linear mixtures of the scattering mechanisms on the ground, in this paper ICA is used to estimate three independent channels, each of them representing a specific scattering mechanism.

\section{The Normalized Compression Distance}

NCD uses the compressed versions of two objects (text strings, documents, voice recordings, images, etc) to compute the degree of similarity between them.

$$
N C D(x, y)=\frac{C(x, y)-\min \{C(x), C(y)\}}{\max \{C(x), C(y)\}}
$$

In this paper NCD is used together with a sliding window to detect the presence of a certain scattering mechanism in the channels estimated using ICA.

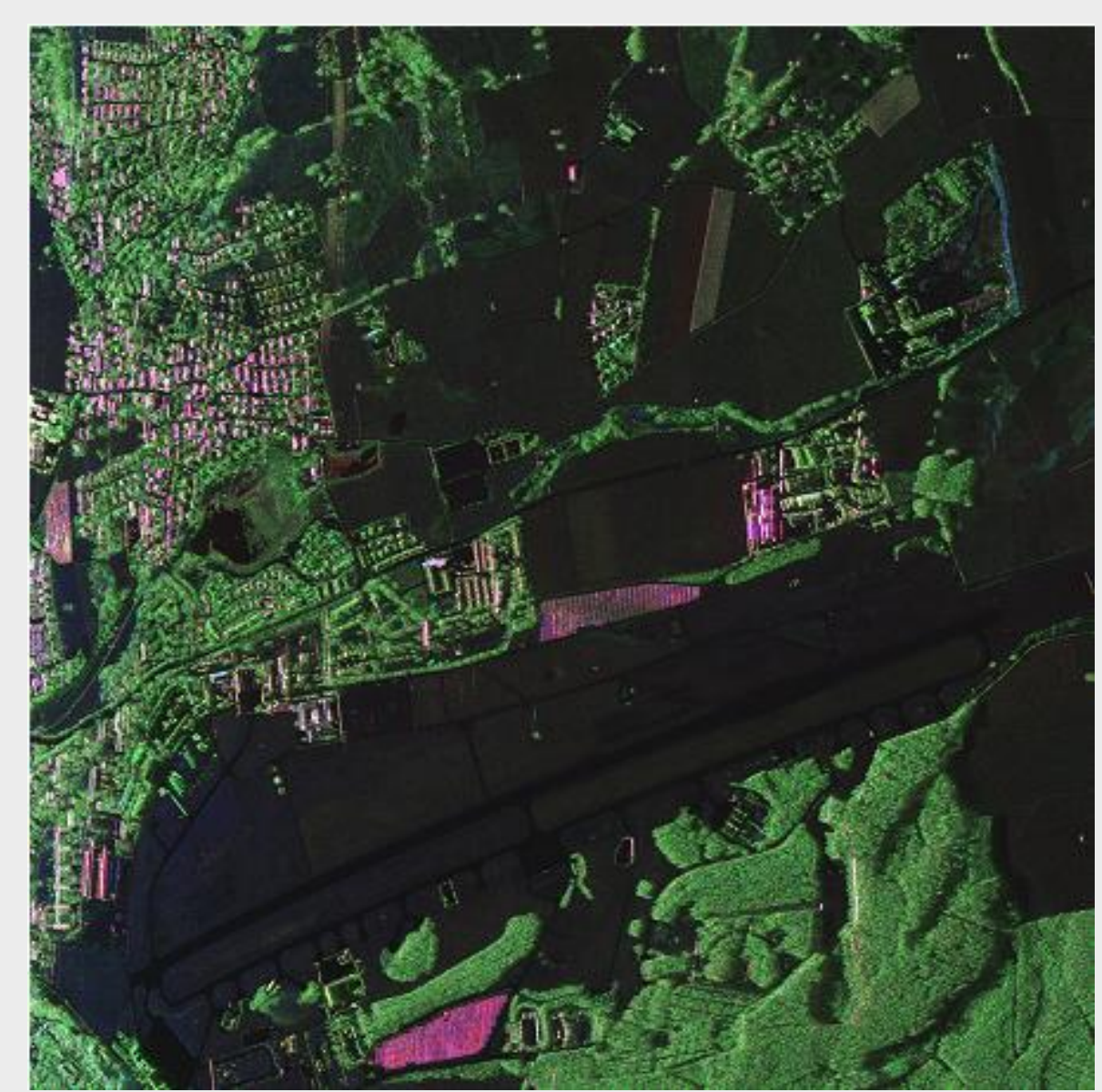

a) Pauli channels

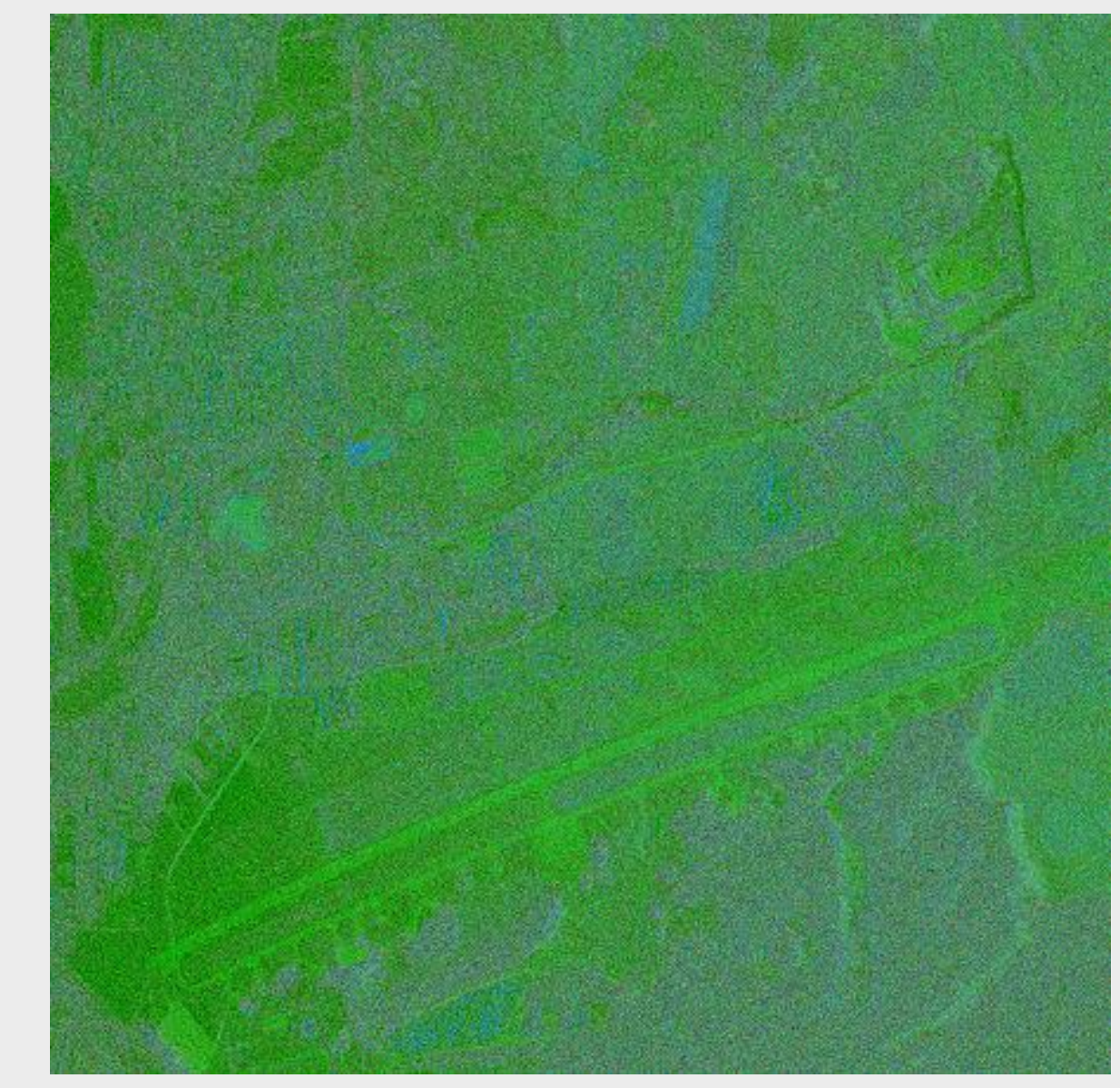

b) H-A- $\alpha$ channels

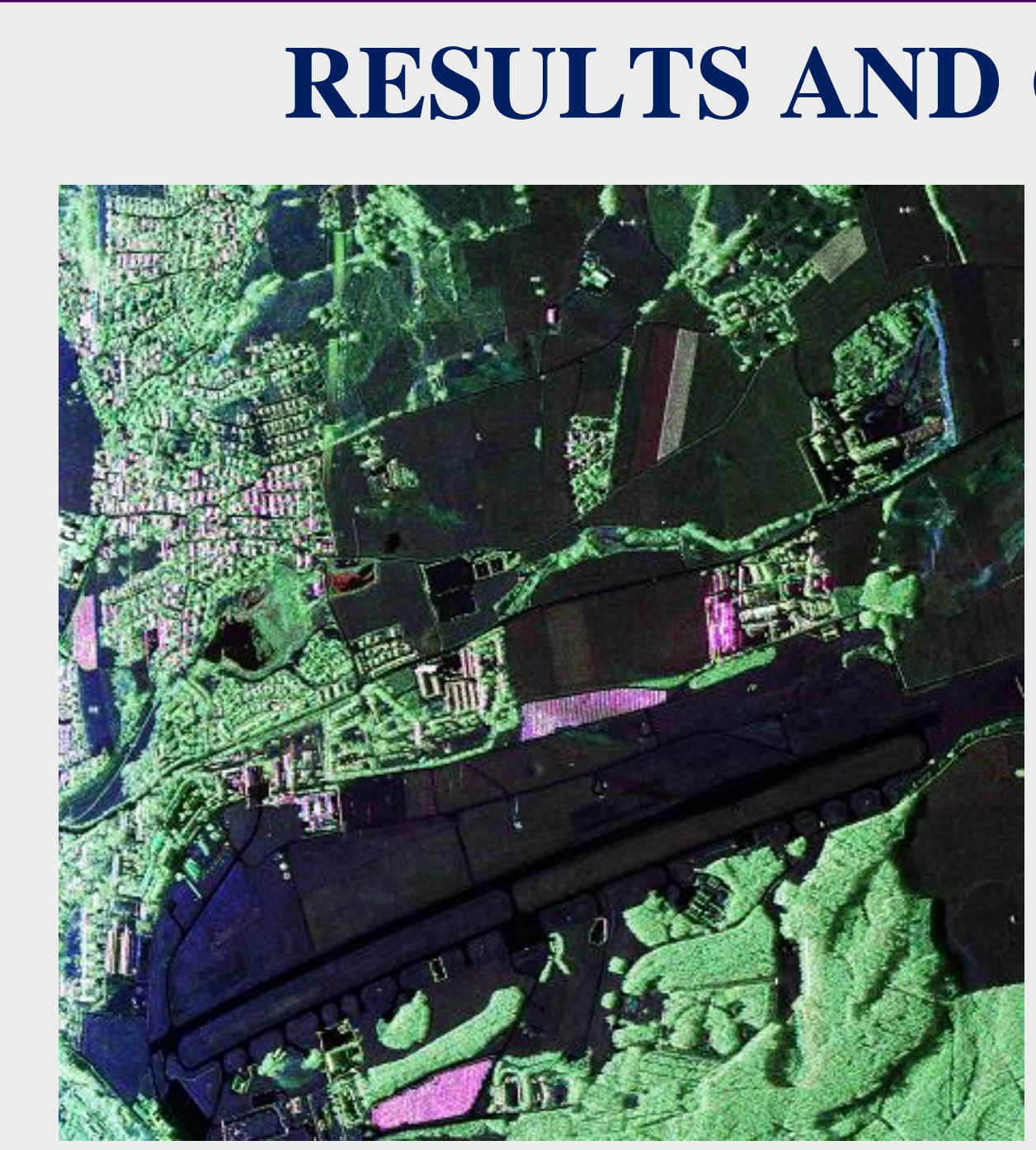

c) Estimated ICA channels

Fig. 1. RGB compositions of the derived channels.

\begin{tabular}{|c|c|c|c|c|c|}
\cline { 2 - 5 } \multicolumn{1}{c|}{} & Urban & Vegetation & Forest & Overall & Mean \\
\hline Pauli 1 & 71.01 & 93.99 & 79.04 & 86.82 & \\
\cline { 1 - 5 } Pauli 2 & 70.53 & 94.09 & 78.30 & 86.61 & \multirow{2}{*}{84.38} \\
\hline Pauli 3 & 29.95 & 94.47 & 70.22 & 79.72 & \\
\hline Entropy & 6.76 & 48.81 & 43.57 & 42.39 & \\
\cline { 1 - 5 } Anisotropy & 7.73 & 82.27 & 0.55 & 48.65 & \multirow{2}{*}{45.94} \\
\hline Alpha & 63.77 & 48.71 & 36.58 & 46.78 & \\
\hline ICA 1 & 70.05 & 95.71 & $\mathbf{8 0 . 7 0}$ & 88.22 & \\
\hline ICA 2 & 29.47 & $\mathbf{9 7 . 3 3}$ & 73.35 & 82.27 & \multirow{2}{*}{84.92} \\
\hline ICA 3 & $\mathbf{7 2 . 9 5}$ & 91.61 & 74.45 & 84.27 & \\
\hline
\end{tabular}

Table 1. Detection rates of the three different scattering mechanisms (urban-double

bounce, low vegetation-single bounce, forest-volume scattering) in the nine channels.

\section{corctasos}
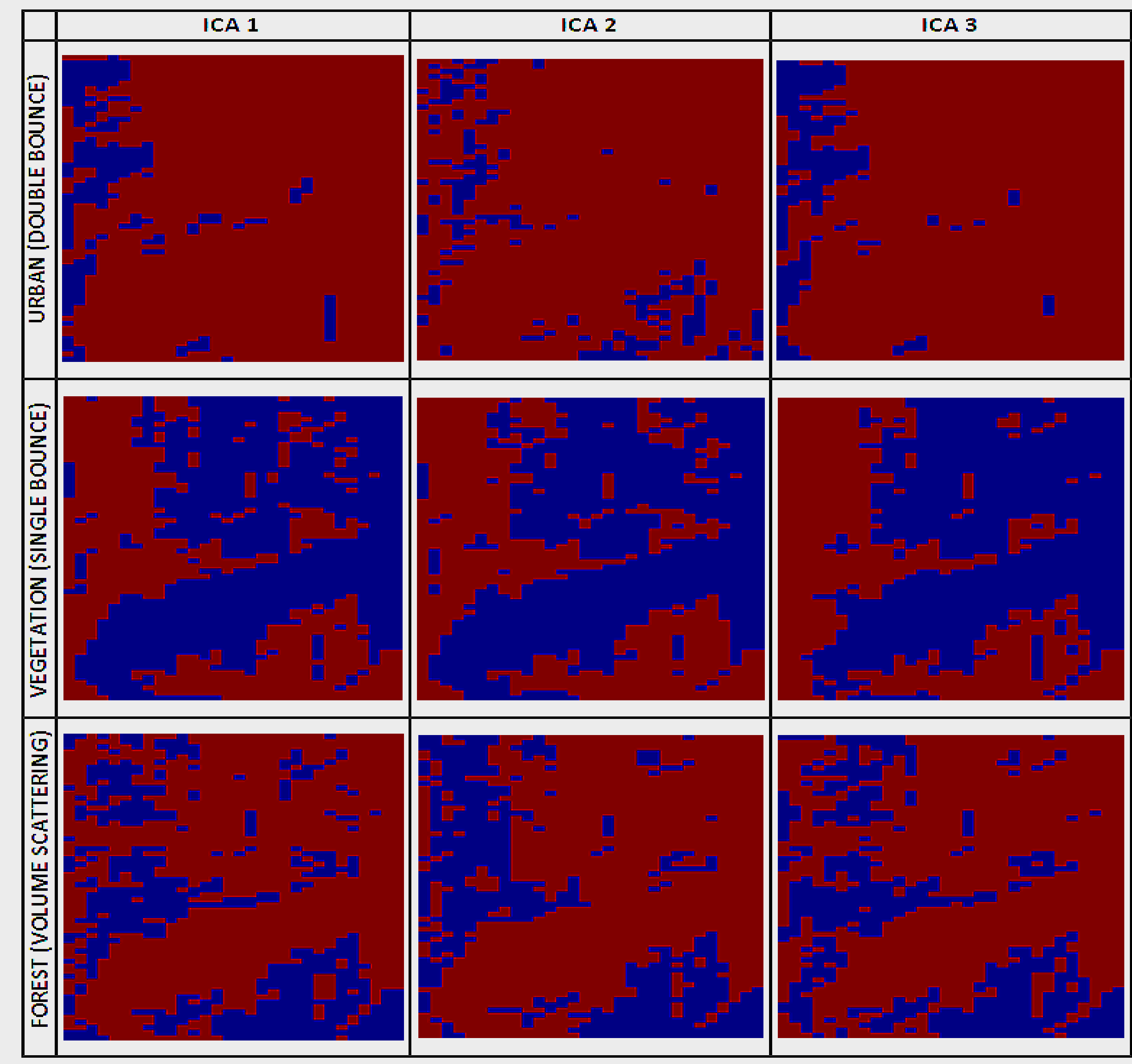

Fig. 2. Graphical representation of the three scattering mechanisms detection results in the three estimated ICA channels. Detected areas are colored in blue.

The best mean overall detection rate was returned by the ICA channels. Pauli channels provided close results, while the H/A/ $\alpha$ channels returned the worst results.

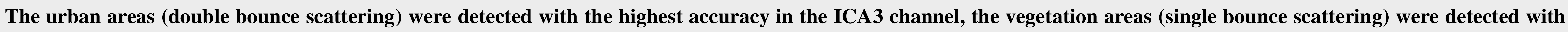

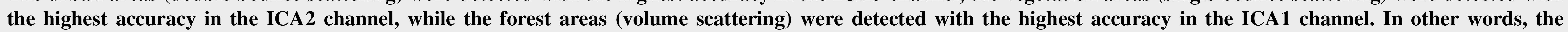
different scattering mechanisms present in the recorded scene were successfully separated by ICA, each one being best detected in a specific ICA channel.

\section{ACKNOWLEDGEMENTS}

- This work has been funded by the Sectoral Operational Programme Human Resources Development 2007-2013 of the Ministry of European Funds through the Financial Agreement POSDRU/159/1.5/S/134398.

- This paper uses data provided by the Microwaves and Radar Institute, German Aerospace Center.

\section{REFERENCES}

S. R. Cloude and E. Pottier, "A Review of Target Decomposition Theorems in Radar Polarimetry," IEEE TGRS, vol. 34, no. 2, pp. 498-518, Mar. 1996.

N. Besic, G. Vasile, J. Chanussot, and S. Stankovic, "Polarimetric Incoherent Target Decomposition by Means of Independent Component Analysis," IEEE TGRS, vol.53, no.3, 2014.

M. Li, X. Chen, Li, B. Ma, and P. M. Vitanyi, "The similarity metric," IEEE Transactions on Information Theory, vol. 50, no. 12, p. 3250-3264, Dec. 2004. 